\title{
To evaluate the profile of patients with disc edema/ papilledema and their presenting pattern
}

\author{
Meena $\mathbf{V}^{1}$, Sharma $\mathbf{U}^{2}$ \\ ${ }^{1}$ Dr. Vandana Meena, Medical officer, Department of Ophthalmology, Gandhi Medical College, Bhopal, MP, ${ }^{2}$ Dr. Unnati \\ Sharma, Ophthalmolgy, GMC, Bhopal, MP, India.
}

Address for Correspondence: Dr. Vandana Meena, Email: meena.vandana@gmail.com

\begin{abstract}
Background: Raised intracranial pressure (ICP) is a potentially life threatening condition, which can also lead to visual loss and blindness. One of the only objective signs accessible during physical examination to confirm a suspicion of raised ICP is papilledema, or swelling of the optic nerve head (ONH). Material \& Methods: The present study is a non randomized prospective case series being conducted in the 50 patients with disc edema/papilledema attending OPD and referred from other departments to DEPARTMENT OF OPHTHALMOLOGY, Gandhi Medical College and associated Hamidia Hospital, Bhopal from January 2013- December 2014. All patients underwent a complete medical evaluation including careful history taking, ophthalmic examination, complete blood count, blood sugar,urea,creatinine, serum lipid profile, thyroid, chest x-ray in specific cases. and CSF analysis (including opening pressure), follow up examination was compared with fundal photography. Results: In our study out of 50 patients, Most commonly affected age group was between 21-30 years in which $32 \%$ cases were observed, $42 \%$ affected were males and $58 \%$ females. Papilledema in $30 \%$ patient were of local cause in which $22 \%$ cases were of optic neuropathy followed by $6 \%$ cases of AION in age group of 51-60 year followed by $2 \%$ cases of BRAO in age group of 41-40 years. Among systemic causes, $24 \%$ cases of ICSOL followed by $12 \%$ cases of meningitis, $10 \%$ cases of malignant hypertension, $8 \%$ cases of drug history, $6 \%$ cases of malaria and $2 \%$ case each of diabetes, pseudotumorcerebri, anaemia, encephalopathy and head injury. Conclusion: ophthalmic evaluation and subjective grading of papilledema by FUNDAL examination has the potential of being a low cost objectively and quantitatively, and assess progression and efficacy of treatment directed toward lowering ICP and thus improve clinical decision-making regarding its diagnosis and treatment papilledema.
\end{abstract}

Keywords: Papilledema, Raised Intracranial Pressure (ICP), Intracranial Hypertension.

\section{Introduction}

Papilledema the acquired bilateral, occasionally asymmetrical, ONH swelling is due to increased intracranial pressure [1]. Papilledema often produces brief episodes of monocular or binocular visual loss, called transient visual obscurations (TVOs) [2,3]. TVOs are often precipitated by postural changes and Valsalvalike maneuvers, with vision rapidly recovering back to baseline between episodes. TVOs are thought to occur due to transient ischemia of the swollen optic nerve head and, although dramatic, are not a sign of impending permanent visual loss. Rarely, IIH patients with papilledema can report gaze-evoked amaurosis, which is transient visual loss precipitated by movement of the eye into an eccentric gaze position [4].

Manuscript received: $22^{\text {nd }}$ Jan 2016

Reviewed: $01^{\text {st }}$ Feb 2016

Author Corrected: $10^{\text {th }}$ Feb 2016

Accepted for Publication: $20^{\text {th }}$ Feb 2016
Untreated papilledema can result in progressive irreversible visual loss and secondary optic atrophy in up to $31 \%$ of patients [2,5,6-8].However, sustained visual loss is less commonly reported than TVOs at the time of presentation [2]. Raised intracranial pressure (ICP) is a potentially life threatening condition, which can also lead to visual loss and blindness [9]. One of the only objective signs accessible during physical examination to confirm a suspicion of raised ICP is papilledema, or swelling of the optic nerve head $(\mathrm{ONH})$. A relatively common cause of papilledema is idiopathic intracranial hypertension, which affects approximately 1 in 100,000 people in the United States and is more prevalent in women with a high body-mass index [10]. Visual field tests may be normal in papilledema, and while medical tests such as lumbar 
puncture and neuroimaging can help in establishing elevated intracranial pressure, they can also give falsepositive and false-negative results[11]. Different imaging modalities have been used to evaluate the topography of $\mathrm{ONH}$ in patients with papilledema $[12,13]$. From monocular color fundus photographs, papilledema can be quantified subjectively using the Frisénscale [14] by taking into account visual features of the optic disc margin and the appearance of discontinuity of blood vessels as they course over the ONH. Stereo disc photography may increase the accuracy of the assessment of papilledema. However, its interpretation is subjective and qualitative and thus depends on the observer's expertise and bias [15]. Early or subtle changes related to papilledema cannot be assessed precisely and objectively owing to the ordinal character of the Frisén scale, which remains one of its limitations, although it is in widespread clinical use. Thus, reliable techniques to assess and quantify papilledema are of high interest and may help the diagnosis and management of disorders causing raised ICP, especially when expertise in ophthalmologic evaluation of the $\mathrm{ONH}$ may not be readily available [11].

Pathogenesis of Papilledema- Because all CSF spaces communicate freely, the pressure andcomposition of the CSF is thought to be the same throughoutthe CNS. Consequently, researchers have based their theoriesand experiments on the assumption that the pressure in theSAS surrounding theONis the same as that in the cerebral andspinal SAS. However, this assumption has not been proved, mainly due to the difficulty in obtaining accurate pressuremeasurements in the SAS of the ON in vivo. In the SAS of theON, CSF flows from the chiasmatic cistern through the canalicularportion and into the intraorbital portion of the $\mathrm{ON}$, aspace that becomes a cul de sac at the back of the globe [16]. Diagnosis of papilledema is made by direct or indirect ophthalmoscopic examination of the optic nerve. Hyperemia of the nerve head, blurring of the optic disc margin, peripapillary flame shaped hemorrhage, dilatation of the capillaries on the surface of nerve \& absence of spontaneous venous pulsation are all clinical signs of papilledema. Nerve fiber layer haemorrhages are the most common finding $\&$ often indicate an acute process [17]. In the present study we will be evaluating the clinical profile of the patients of disc edema with special reference to Papilledema to highlight the role and significance of fundus evaluation at various stages and it's clinical correlation.

\section{Material \& Methods}

The present study is a non randomized prospective case series being conducted in the patients with disc edema/papilledema attending OPD and referred from other departments to RIO, DEPARTMENT OF OPHTHALMOLOGY, Gandhi Medical College and associated Hamidia Hospital, Bhopal from January 2013- December 2014.

\section{Inclusion Criteria:}

- Proven case of disc edema

- Proven case of papilledema

Exclusion Criteria:

- Cases of pseudopapilledema

All patients underwent a complete medical evaluation including careful history taking, ophthalmic examination, complete blood count, blood sugar, urea, creatinine, serum lipid profile, thyroid, chest $\mathrm{x}$-ray in specific cases. and CSF analysis (including opening pressure). Ocular examination consists of visual acuity measurement with Snellen's chart, anterior segment examination using slit lamp biomicroscopy, applanation tonometry, stereoscopic fundus photography and visual fields evaluation using automated perimetry with the Humphrey 30-2 program. The degree of papilledema was graded using Frisen's scheme [3,4].

Thorough ophthalmic examination including

a. Assessment of visual acuity on Snellen's chart and near vision.

b. Relative afferent pupillary defect- by swinging flash torch light examination.

c. Colour vision by Ishihara's chart

3. Intra ocular pressure-recorded with Schiotz indentation tonometer

4. Slit lamp examination.

5. Optic disc evaluation.

Using +78D/90D Condensing lens.

Following details were noted

DISC

Cup size-

Color of the disc-

Cup disc ratio-

Cup disc asymmetry between two eyes-

Blurring of the disc margins-

Hemorrhage over disc-

Tortuosity of vein-

Venous pulsation- 
In normal eye venous pulsation is present, if it is absent and not appear on pressing the globe it is indication of papilledema.Fundus evaluation with indirect ophthalmoscope and $+90 \mathrm{D}$ slit lamp examination done. In early papilledema we may get following fundus findings.

a) Hyperemia of the disc

b) Blurring of the disc margin

c) Apparent forward protrusion of disc

d) Blurring of the physiological cup

e) Overfilling of the vein

f) In some early case of papilledema, haemorrhage and exudates may be present at some distance from the disc. Fully developed and late papilledema. The physiological cup becomes partially or completely obliterated, the margin of the disc becomes definitely blurred, the surrounding retina may have grayish tinge and the vessels are seen to climb to attain the disc, the veins become engorged. Haemorrhage may appear as a linear streak on the disc or around it. Persistent papilledema: The arteries are not any time narrowed because when the arteries exhibit narrowing atrophic changes in the disc invariably follow. Visual acuity, optic disc changes, and visual field defects were checked in all the patients during follow-up, which was done every 2 weeks for a month, monthly for 3 months, and after that every 6 months for 2 years.

Statistical Analyses: The analyses were largely descriptive, with means, standard deviations, and ranges reported for continuous variables and counts and percentages reported for categorical variables. Associations between continuous variables are described using either Pearson correlation coefficients or Spearman rank correlation coefficients, as appropriate.

\section{Results}

Table 1: Age distribution

\begin{tabular}{|l|l|l|}
\hline Age & No of Patient $(\mathbf{n = 5 0})$ & \% of Patient \\
\hline O-10 & 06 & 12 \\
\hline $11-20$ & 12 & 24 \\
\hline $21-30$ & 16 & 32 \\
\hline $31-40$ & 8 & 16 \\
\hline $41-50$ & 5 & 10 \\
\hline $51-60$ & 3 & 6 \\
\hline Total & $\mathbf{5 0}$ & $\mathbf{1 0 0}$ \\
\hline
\end{tabular}

Most commonly affected age group was between 21-30 years in which $32 \%$ case were observed, least common affected age group was 51-60 years in which $6 \%$ case were observed.

\section{Table 2: Sex distribution}

\begin{tabular}{|l|l|l|}
\hline Sex & No of Case $(\mathbf{n}=\mathbf{5 0})$ & \% of Case \\
\hline Male & 21 & 42 \\
\hline Female & 29 & 58 \\
\hline Total & $\mathbf{5 0}$ & $\mathbf{1 0 0}$ \\
\hline
\end{tabular}

In this study $42 \%$ affected were males and $58 \%$ females.

Table-3: Laterality

\begin{tabular}{|l|l|l|}
\hline Laterality of Disc Edema & No of Case $(\mathbf{n}=\mathbf{5 0})$ & \% of Case \\
\hline Unilateral & 15 & 30 \\
\hline Bilateral & 35 & 70 \\
\hline Total & $\mathbf{5 0}$ & $\mathbf{1 0 0}$ \\
\hline
\end{tabular}

In this study $70 \%$ cases of bilateral (papilloedema) and $30 \%$ case of unilateral disc edema were observed. 
Table-4: Local Causes

\begin{tabular}{|l|l|l|l|}
\hline & Causes & No of Case & \% of case \\
\hline \multirow{4}{*}{ Local Causes } & Optic neuropathy & 11 & 22 \\
\cline { 2 - 4 } & Aion & 3 & 6 \\
\cline { 2 - 4 } & Brao & 1 & 2 \\
\cline { 2 - 4 } & Hypotony & 0 & 0 \\
\cline { 2 - 4 } & Raised iop & 0 & 0 \\
\hline Total & $\mathbf{1 5}$ & $\mathbf{3 0}$ \\
\hline
\end{tabular}

In this study out of 50 patient, $30 \%$ patient were of local cause in which $22 \%$ cases were of optic neuropathy followed by $6 \%$ cases of AION in age group of 51-60 year followed by 2\% cases of BRAO in age group of 41-40 years.

Table-5: Systemic Causes

\begin{tabular}{|l|l|l|l|}
\hline Systemic Causes & Causes & No of Cases & \% of Cases \\
\cline { 2 - 4 } & Icsol & 12 & 24 \\
\cline { 2 - 4 } & Diabetes & 1 & 2 \\
\cline { 2 - 4 } & Malignant hypertension & 5 & 10 \\
\cline { 2 - 4 } & Meningitis & 6 & 12 \\
\cline { 2 - 4 } & Drug history & 4 & 8 \\
\cline { 2 - 4 } & Pseudo tumor cerebri & 1 & 2 \\
\cline { 2 - 4 } & Malaria & 3 & 6 \\
\cline { 2 - 4 } & Anaemia & 1 & 2 \\
\cline { 2 - 4 } & Encephalopathy & 1 & 2 \\
\cline { 2 - 4 } & Head injury & 1 & 2 \\
\cline { 2 - 4 } & Total & $\mathbf{3 5}$ & $\mathbf{7 0}$ \\
\hline
\end{tabular}

In this study out of 50 patient, $24 \%$ cases of ICSOL in systemic causes followed by $12 \%$ cases of meningitis, $10 \%$ cases of malignant hypertension, $8 \%$ cases of drug history, $6 \%$ cases of malaria and $2 \%$ case each of diabetes, pseudotumorcerebri, anaemia , encephalopathy and head injury.

Table-6 Symptoms of disc edema/papilledema

\begin{tabular}{|l|l|l|}
\hline Symptom & No of C`ases & \% of Cases \\
\hline Dov & 25 & 50 \\
\hline Headache & 35 & 70 \\
\hline Nausia/vomiting & 24 & 48 \\
\hline Transient obscuration of vision & 8 & 16 \\
\hline Lr palsy & 2 & 4 \\
\hline Diplopia & 2 & 4 \\
\hline
\end{tabular}

Most common presenting complaint was headache in $70 \%$ cases followed by DOV in $50 \%$ cases, nausea and vomiting in $48 \%$ cases, LR palsy and Diplopia in $4 \%$ cases.

\section{Discussion}

Papilloedema simply means oedema of the opticdisc, without reference to its underlying cause. Recognition of papilloedema is of great clinicalimportance because it is the classic and mostimportant clinical sign of raised intracranialpressure. Modern diagnostic methods haveadvanced to such an extent that the recognitionand treatment of raised intracranial pressure isusually 
undertaken before papilloedemaappears [18]. Among symptomatology, headache is the most common symptom in our study similar study is by Julayanont $P$ et al[19]they studied, idiopathic intracranial hypertension (IIH) is an uncommon disorder characterized by increased intracranial pressure without radiological or laboratory evidence of intracranial pathology except empty sellaturcica, optic nerve sheath with filled out cerebrospinal fluid spaces, and smooth-walled nonflow-related venous sinus stenosis or collapse. This condition typically affects obese women. The incidence of $\mathrm{IIH}$ is increasing with the rising prevalence of obesity. Persistent headache is the most common symptom. Visual impairment is a serious complication that may not be recognized by the patients. This paper reviews clinical manifestations, diagnostic challenges, and current treatments of $\mathrm{IH}$ in adults. Various imaging modalities have been studied on their validity for detection of $\mathrm{IH}$ and papilledema. This review also includes new studies on medical, surgical, and interventional management of this condition [19].

Compression of optic nerve and optic nerve ischemia are the two major theories explaining papilledema caused by elevated ICP in IIH [20]. Papilledema is one of the hallmarks for diagnosis of IIH. Even though, papilledema is commonly symmetric or only mildly asymmetric, significant asymmetry may be found in some patients and can be explained by difference in size of bony optic canals or variation of trabecular meshwork in subarachnoid space surrounding optic discs [21,22]. Unilateral papilledema is not commonly reported [23,24], similar to our study.

Fundoscopic examination is very important to evaluate this condition. Frisén's [14] criteria has been widely accepted for grading severity of papilledema. It is very important to differentiate true papilledema from pseudoedema, which is the physiologic variant, or benign changes of the optic disc. Findings from funduscopic examination showed that the presence of retinal or choroidal folds is a pathognomonic sign of true papilledema. However, this finding was seen only in $23 \%$ of the patients with true papilledema. The combination of four features from funduscopicexamination, including the swelling of the peripapillary retinal nerve fiber layer, peripapillary hemorrhages, papilla elevation, and congestion of peripapillary vessels, provides very good validity in differentiating true papilledema from pseudoedema (accuracy 93\%, sensitivity 95\%, and specificity $89 \%$ )[25]. There is a study aimed at identifying the CSF proteome in IIH patients as the new biomarkers. Six proteins were upregulated in $\mathrm{IIH}$, namely, sterol regulatory element-binding protein 1 , zinc- $\alpha-2$ glycoprotein, immunoglobulin heavy constant $\alpha-1, \alpha-1$ antitrypsin, serotransferrin, and haptoglobin. Four proteins were downregulated in IIH, including hemopexin, angiotensinogen, vitamin-D-binding protein, and transthyretin. Angiotensinogen was the first protein validated in the study, and it was found that down-regulation of angiotensinogen may contribute to the increased CSF production, which subsequently causes IIH. The study of other proteins may provide more knowledge on the new biomarkers for diagnosis of IIH. Moreover, these proteins may be the target for therapeutic intervention [26].

As expected, the majority of patients in this series reported headaches, consistent with previous studies $[27,28,29]$. Depression was present in about half the patients in both groups; this finding is also consistent with previous studies [30]. Pulsatile tinnitus was a common symptom in both IIHWP patients $(48 \%)$ and IIHWOP patients (33\%); this was consistent with previous findings [28]. A significant number of IIHWOP patients in this series reported auras. This may indicate that a significant number of IIHWOP patients are also migraine sufferers. Indeed, it has been observed that most patients with IIH have migrainous headaches unrelated to increased intracranial pressure [31]. Some may argue that IIHWOP is merely migraine in obese individuals. However, all of our IIHWOP patients underwent carefully performed measurements of OPs or they were not included in the study. In addition, $65 \%$ of our patients had 2 or more elevated reading on more than one occasion. Furthermore, 11 of our patients with IIHWOP had one or more other symptoms of increased ICP (besides headache). Finally, 4 of our IIHWP patients also had aura symptoms with migraine in addition to intracranial hypertension, headache is also common symptom in our study. Vertigo was more frequently present in IIHWOP although nearly half of the IIHWP subjects also endorsed dizziness or vertigo on a review of systems. In a study reviewing complaints of patients with $\mathrm{IIH}$, vertigo and dizziness are more common when clinicians routinely query for them [32]. Children with IIH frequently complain of dizziness and are ataxic [33]. In the recent William F. Hoyt Lecture of the American Academy of Ophthalmology, Dr Jonathan Trobe posited that 
papilledema is only a reliable indicator of chronically high ICP because the development of papilledema tends to lag behind the rise in ICP. Trobe noted that fewer than $20 \%$ of patients examined within a few days of head trauma or ruptured aneurysm have papilledema and only $6 \%$ of patients with chronically high ICP lack papilledema [34].

In this study out of 50 patient, $30 \%$ patient were of local cause in which $22 \%$ cases were of optic neuropathy followed by $6 \%$ cases of AION in age group of 51-60 year followed by $2 \%$ cases of BRAO in age group of 41-40 years. $24 \%$ cases of ICSOL in systemic causes followed by $12 \%$ cases of meningitis, $10 \%$ cases of malignant hypertension, $8 \%$ cases of drug history, $6 \%$ cases of malaria and $2 \%$ case each of diabetes, pseudotumorcerebri, anaemia , encephalopathy and head injury,similarly Agrawal et al[18] found systemic causes are more prominent than local causes, in which ICSOL are most common among all causes.

In this study $70 \%$ cases of bilateral (papilloedema) and $30 \%$ case of unilateral disc edema were observed which is correlated with many studies[2,3,4] while Bidot $\mathrm{S}$ et al[21] studied, asymmetric papilledema in idiopathic intracranial hypertension (IIH) and found Of the 559 adult patients with definite IIH, 20 (3.6\%; 95\%CI: 2.3$5.6 \%)$ had very asymmetric papilledema at initial evaluation. They were older (39 versus 30 years; $\mathrm{p}<0.001$ ), had lower cerebrospinal opening pressure ( 35.5 versus $36 \mathrm{~cm}$ of water; $\mathrm{p}=0.03$ ), and were more likely to be asymptomatic compared to patients with symmetric papilledema ( $27 \%$ versus $3 \%$; $<<0.001)$. Visual fields were worse on the side of the highestgrade papilledema $(\mathrm{p}=0.02)$. The bony optic canal was smaller on the side of the lowest-grade edema in all 8 patients $(100 \%)$ in whom the imaging was sufficient for reliable measurements $(\mathrm{p}=0.008)$.

\section{Conclusion}

Because ophthalmoscopic evaluation and subjective grading of papilledema can show significant variability among observers and requires specialized clinical expertise, the estimate of $\mathrm{ONH}$ shape and quantification of optic disc swelling from stereo fundus photographs by three-dimensional (3-D) image analysis methodology may improve reproducibility and reliability of the assessment of papilledema and thus improve clinical decision-making regarding its diagnosis and treatment. Our study does not speak to the clinical profile and treatment of papilledema in all cases; evidence-based treatments of these patients await future randomized clinical trials.

Funding: Nil,

Conflict of interest: None.

Permission of IRB: Yes

\section{References}

1. Friedman, D, Jacobson, D. Idiopathic Intracranial Hypertension. J. Neuro-Ophthal2004; 59: 1492-1495.

2. Wall M, George D. Idiopathic intracranial hypertension. A prospective study of 50 patients. Brain. 1991 Feb;114 ( Pt 1A):155-80.

3. Corbett JJ. The first Jacobson Lecture. Familial idiopathic intracranial hypertension. J Neuroophthalmol. 2008 Dec;28(4):337-47. doi: 10.1097/WNO.0b013e31818f12a2.

4. O'Duffy D, James B, Elston J. Idiopathic intracranial hypertension presenting with gaze-evoked amaurosis. Acta Ophthalmol Scand. 1998 Feb;76(1):119-20.

5. Corbett JJ, Savino PJ, Thompson HS, Kansu T, Schatz NJ, Orr LS, Hopson D. Visual loss in pseudotumorcerebri: follow-up of 57 patients from five to 41 years and a profile of 14 patients with permanent severe visual loss. Arch Neurol. 1982;39:461474.http://www.ncbi.nlm.nih.gov/pubmed

6. Orcutt JC, Page NG, Sanders MD. Factors affecting visual loss in benign intracranial hypertension.

Ophthalmology. 1984 Nov;91(11):1303-12.

7. Radhakrishnan K, Thacker AK, Bohlaga NH, Maloo JC, Gerryo SE. Epidemiology of idiopathic intracranial hypertension: a prospective and case-control study. J Neurol Sci. 1993 May;116(1):18-28.

8. Rowe FJ, Sarkies NJ. Assessment of visual function in idiopathic intracranial hypertension: a prospective study. Eye (Lond). 1998;12 ( Pt 1):111-8.

9. Cameron AJ. Marked papilloedema in pulmonary emphysema. Br J Ophthalmol. 1933 Mar;17(3):167-9.

10. Binder DK, Horton JC, Lawton MT, McDermott MW. Idiopathic intracranial hypertension. Neurosurgery. 2004 Mar;54(3):538-51; discussion 5512. 
11. Menke MN, Feke GT, Trempe CL. OCT measurements in patients with optic disc edema. Invest Ophthalmol Vis Sci. 2005 Oct;46(10):3807-11.

12. Trick GL, Vesti E, Tawansy K, Skarf B, Gartner J. Quantitative evaluation of papilledema in pseudotumor cerebri. Invest Ophthalmol Vis Sci. 1998 Sep;39(10):1964-71.

13. Rebolleda G, Munoz-Negrete FJ. Follow-up of mild papilledema in idiopathic intracranial hypertension with optical coherence tomography. Invest Ophthalmol Vis Sci. 2009;50:5197-5200.

14. Frisén L. Swelling of the optic nerve head: a staging scheme. J Neurol Neurosurg Psychiatry. 1982 Jan;45(1):13-8.

15.Scott CJ, Kardon RH, Lee AG, Frisen L, Wall M. Diagnosis and grading of papilledema in patients with raised intracranial pressure using optical coherence tomography vs clinical expert assessment using a clinical staging scale. Arch Ophthalmol. 2010;128:705711

16. Killer HE, Jaggi GP, Miller NR. Papilledema revisited: is its pathophysiology really understood? Clin Experiment Ophthalmol. 2009 Jul;37(5):444-7. doi: 10.1111/j.1442-9071.2009.02059.x.

17.Friedman DI. Papilledema. In: Miller NR, Newman NJ. Walsh and Hoyt's Clinical Neuro-Ophthalmolgy, 6th Ed.Baltimore: Lippincott Williams and Wilkins, 2005: 237-291

18.Agarwal, A, Yadav P. Papilledema (choked disc) Journal, Indian Academy of Clinical Medicine _ Vol. 1, No. 3 _ October-December 2000.

19. Julayanont $P$, Karukote $A^{2}$, Ruthirago $D^{1}$, Panikkath $\mathrm{D}^{3}$, Panikkath $\mathrm{R}^{3}$. Idiopathic intracranial hypertension: ongoing clinical challenges and future prospects. J Pain Res. 2016 Feb 19;9:87-99. doi: 10.2147/JPR.S60633. eCollection 2016.

20. Passi N, Degnan AJ, Levy LM. MR imaging of papilledema and visual pathways: effects of increased intracranial pressure and pathophysiologic mechanisms. AJNR Am J Neuroradiol. 2013 May;34(5):919-24. doi: 10.3174/ajnr.A3022. Epub 2012 Mar 15.
21. Bidot S, Bruce BB, Saindane AM, Newman NJ, Biousse V. Asymmetric papilledema in idiopathic intracranial hypertension. J Neuroophthalmol. 2015 Mar;35(1):31-6. doi: 10.1097/WNO.0000000000000205.

22. Maxner CE, Freedman MI, Corbett JJ. Asymmetric papilledema and visual loss in pseudotumour cerebri. Can J Neurol Sci. 1987 Nov;14(4):593-6.

23. Brosh K, Strassman I. Unilateral papilledema in pseudotumor cerebri. Semin Ophthalmol. 2013 Jul;28(4):242-3. doi: 10.3109/08820538.2013.768677. Epub 2013 Apr 29.

24. Wattamwar PR, Baheti NN, Radhakrishnan A. Idiopathic intracranial hypertension presenting as unilateral papilledema. Neurol India. 2010 SepOct;58(5):818-9. doi: 10.4103/0028-3886.72208.

25. Carta A, Favilla S, Prato M, Bianchi-Marzoli S, Sadun AA, Mora P. Accuracy of funduscopy to identify true edema versus pseudoedema of the optic disc. Invest Ophthalmol Vis Sci. 2012 Jan 3;53(1):1-6. doi: 10.1167/iovs.11-8082.

26. Brettschneider J, Hartmann $\mathrm{N}$, Lehmensiek V, Mogel H, Ludolph AC, Tumani H. Cerebrospinal fluid markers of idiopathic intracranial hypertension: is the renin-angiotensinogen system involved? Cephalalgia.2011;31(1):116121.http://www.ncbi.nlm.nih.gov/pubmed [PubMed]

27. Digre KB, Corbett JJ. Idiopathic intracranial hypertension (pseudotumorcerebri): A reappraisal.Neurologist. 2001;7:2-67.

28. Wang SJ, Silberstein SD, Patterson S, et al. Idiopathic intracranial hypertension without papilledema: A case-control study in a headache center. Neurology. 1998;51:245-249.

29. Vieira DS, Masruha MR, Goncalves AL, et al. Idiopathic intracranial hypertension with and without papilloedema in a consecutive series of patients with chronic migraine. Cephalalgia. 2008;28:609-613.

30. Kleinschmidt JJ, Digre KB, Hanover R. Idiopathic intracranial hypertension: Relationship to depression, anxiety, and quality of life. Neurology. 2000;54:319324. 
31. Friedman DI, Rausch EA. Headache diagnoses in patients with treated idiopathic intracranial hypertension. Neurology. 2002 May 28;58(10):1551-3.

32. Murphy TP. Otologic manifestations of pseudotumor cerebri. J Otolaryngol. 1991;20:258261.http://www.ncbi.nlm.nih.gov/pubmed[PubMed]
33. Rangwala LM, Liu GT. Pediatric idiopathic intracranial hypertension. Surv Ophthalmol. 2007 NovDec;52(6):597-617.

34. Trobe JD. Papilledema: the vexing issues. J Neuroophthalmol. $2011 \quad$ Jun;31(2):175-86. doi: 10.1097/WNO.0b013e31821a8b0b.

\section{How to cite this article?}

Meena V, Sharma U To evaluate the profile of patients with disc edema/ papilledema and their presenting pattern. Int J Med Res Rev 2016;4(3):301-308. doi: 10.17511/ijmrr.2016.i03.03. 